\title{
Nuevos Retos en el Tratamiento del Juego Patológico
}

\section{New Challenges in the Treatment of Disordered Gambling}

\author{
Enrique Echeburúa, \\ Karmele Salaberría \\ Marisol Cruz-Sáez \\ Universidad del País Vasco (UPV/EHU), España
}

(Rec: 30 mayo 2013 / Acept: 16 septiembre 2013)

\begin{abstract}
Resumen
En este artículo se señalan los avances y retos de futuro en el tratamiento del juego patológico. El objetivo terapéutico (abstinencia o juego moderado) es actualmente objeto de controversia. Los tratamientos para la ludopatía incluyen la terapia hospitalaria, los tratamientos cognitivo-conductuales individuales o grupales, Jugadores Anónimos y la farmacoterapia, así como la prevención de recaídas. La terapia cognitivo-conductual presenta unas tasas de éxito del $50 \%$ al $80 \%$ de los casos tratados en un seguimiento a largo plazo. La farmacoterapia es un complemento cuando los pacientes tienen un estado de ánimo deprimido o un elevado nivel de impulsividad. El juego controlado puede ser una alternativa terapéutica para los jugadores jóvenes o que no presentan aún una dependencia severa. Se requiere más información sobre el tratamiento del juego on-line y de poblaciones específicas (mujeres y jóvenes). Se comentan las implicaciones de esta revisión para la práctica clínica y para las investigaciones futuras.

Palabras clave: Juego patológico, objetivos terapéuticos, tratamiento psicológico, terapia farmacológica, prevención de recaídas.
\end{abstract}

\begin{abstract}
This paper deals with the new developments in the treatment of disordered gambling, as well as with the challenges for further research. Abstinence versus moderated gambling is an issue that raises many concerns and that needs to be addressed. Current treatment for disordered gambling involves a number of different options, including inpatient treatments, individual and group cognitive-behavioral options, Gamblers Anonymous and pharmacotherapy, as well as an intervention in relapse prevention. Cognitive-behavioral therapy may have asuccess rate ranging from $50 \%$ to $80 \%$ of treated patients in a long-term follow-up. Psychopharmacological therapy may have incremental benefit when patients have comorbid depression or high impulsivity. Responsible gambling may be a therapeutic option for young gamblers or people without a severe dependence. Further information is required about treatment for online gambling addictions and for dealing with specific populations (women and young people). Unanswered questions for future research in this field are commented upon.

Keywords:Disordered gambling, therapeutic goals, psychological treatment, pharmacological therapy, relapse prevention.
\end{abstract}

Correspondencia: Enrique Echeburúa. Facultad de Psicología. Universidad del País Vasco. Avda. de Tolosa, 70. 20018 San Sebastián (España). E-mail: enrique.echeburua@ehu.es

Los autores pertenecen al Grupo Consolidado de Investigación en Psicología Clínica del Gobierno Vasco y a la UFI 11/04 de la Universidad del País Vasco (UPV/EHU). 


\section{Introducción}

La ludopatía es una forma de adicción sin drogas. Las adicciones, tal como figura en la nueva edición del DSM5 (American PsychiatricAssociation, 2013), no se limitan exclusivamente a las conductas problemáticas generadas por algunas sustancias químicas, como los opiáceos, los estimulantes, los ansiolíticos, la nicotina o el alcohol. Lo cierto es que de conductas normales, como implicarse en juegos de apuestas, conectarse a Internet o recurrir a las redes sociales, se pueden hacer usos anormales en función de la intensidad, de la frecuencia o de la cantidad de dinero invertida $\mathrm{y}$, en último término, en función del grado de interferencia negativa en las relaciones familiares, sociales y laborales/ académicas de las personas implicadas (Echeburúa y Corral, 2008). En la tabla 1 figuran los criterios del DSM-5 para el juego patológico, que, de momento, es la única adicción sin droga considerada como tal (el-Guebaly, Mudry, Zohar, Tavares y Potenza, 2012).

Tabla 1.Criterios diagnósticos del juego patológico en el DSM5 (American PsychiatricAssociation, 2013)

El paciente muestra una conducta de juego persistente y desadaptativa, tal como se refleja en la presencia de 4 o más de los siguientes síntomas:

1) Preocupación por el juego.

2) Necesidad de jugar con cantidades crecientes de dinero para conseguir el grado de excitación deseado.

3) Fracasos repetidos en los esfuerzos para controlar el juego.

4) Inquietud o irritabilidad cuando se intenta interrumpir o detener el juego.

5) Utilización del juego como vía de escape de los problemas o de alivio del malestar emocional.

6) Intentos repetidos de recuperar el dinero perdido.

7) Mentiras a la familia y a los terapeutas sobre la implicación en el juego.

8) Puesta en peligro o pérdida de relaciones personales significativas, de trabajo o de oportunidades educativas debido al juego.

9) Apoyo económico reiterado por parte de la familia y de los amigos.

Las adicciones sin drogas funcionan, en unos casos, como conductas sobreaprendidas que traen consigo consecuencias negativas y se adquieren a fuerza de repetir comportamientos que en un principio resultan agradables; y en otros, como estrategias de afrontamiento inadecuadas para hacer frente a los problemas personales (por ejemplo, acudir al bingo para hacer frente a la ansiedad o a la tristeza).
La presencia incontrolada de máquinas tragaperras en bares y lugares de ocio, junto con la oferta abundante de bingos, casinos y de los juegos más tradicionales (quinielas, loterías, cupones, carreras de caballos, etcétera), así como de las casas de apuestas y del juego on-line, ha hecho aumentar considerablemente la ludopatía. La indefensión ante esta avalancha de juegos con apuestas afecta especialmente a los adolescentes y a las personas más vulnerables psicológicamente.

En España, la tasa de prevalencia de la ludopatía, incluyendo en ella la dependencia al juego y el juego problemático, oscila entre el $2 \%$ y el $3 \%$ de la población adulta (Becoña, 1999). Estos datos son similares a los obtenidos en otros países (Lorains, Cowlissahaw y Thomas, 2011). El juego patológico implica la pérdida de control, la dependencia emocional, la tolerancia y la interferencia grave en la vida cotidiana; los jugadores problemáticos, a su vez, se caracterizan por presentar dificultades en el funcionamiento diario (manejo del dinero, deudas contraídas, mentiras, aislamiento social, relaciones con la pareja, etcétera) debidas al juego, pero sin mostrar (todavía) los criterios diagnósticos de la ludopatía.

El trastorno es mucho más frecuente en hombres que en mujeres, pero estas son mucho más reacias a buscar ayuda terapéutica por la censura social existente. Mientras las motivaciones de los hombres para jugar son la obtención de un dinero fácil y la búsqueda de excitación, las mujeres juegan más para aliviar el malestar emocional y para hacer frente a la soledad. Las mujeres comienzan a jugar a una edad más tardía que los hombres, pero la adicción se desarrolla más rápidamente. Sin embargo, estas diferencias no se han tenido en cuenta a la hora de diseñar tratamientos específicos. De hecho, son muy pocas las mujeres que han formado parte de las investigaciones clínicas publicadas hasta la fecha (Echeburúa, González-Ortega, Corral y Polo-López, 2011; González-Ortega, Echeburúa, Corral, Polo-López y Alberich, 2013).

A diferencia de otras conductas adictivas, el juego patológico se distribuye por todas las clases sociales y por todas las edades. No obstante, la edad de acceso al juego ha descendido en los últimos años. De este modo, cada vez son más los adolescentes que buscan tratamiento por problemas de juego.

Desde una perspectiva psicopatológica, la depresión, los trastornos de ansiedad y el abuso de alcohol están asociados con frecuencia al juego patológico en forma de trastornos comórbidos. Así, la tasa de prevalencia del consumo abusivo de alcohol u otras drogas entre los ludópatas oscila alrededor del 15\%-20\% (Lorains et al., 2011). 


\section{Objetivos terapéuticos}

No se ha abordado de una forma rigurosa la cuestión del objetivo terapéutico en el tratamiento de la ludopatía. Tradicionalmente, y por influencia de Jugadores Anónimos y de los programas terapéuticos llevados a cabo con el alcoholismo y otras toxicomanías, la meta del tratamiento ha sido la abstinencia total (Ladouceur, 2005).

Sin embargo, se ha comenzado recientemente a reconsiderar la abstinencia como único objetivo terapéutico para los ludópatas. Lo que ha llevado a plantear otras metas (el juego controlado) es la escasa motivación para el tratamiento constatada en muchos ludópatas (solo un 10\% de los sujetos identificados como ludópatas en los estudios epidemiológicos están en tratamiento), así como la constatación de que hay ludópatas tratados con éxito que juegan esporádicamente sin recaer en la ludopatía. Se trata, por ello, de ofrecer una opción de tratamiento que resulte más sugerente para atraer pacientes a la terapia, mantenerlos en el tratamiento y evitar un mayor número de recaídas. Sin embargo, los resultados empíricos obtenidos hasta la fecha con este objetivo son aún inconsistentes y no parecen superar los logrados con los programas de abstinencia.

El problema de los programas de juego controlado es que no se define propiamente el concepto de juego controlado ni tampoco se precisa con claridad el tipo de pacientes a los que van destinados. Por lo que se refiere al primer punto, es imprescindible detallar lo que se entiende exactamente por juego controlado en relación con los siguientes parámetros: a) pautas de juego (límites de tiempo invertido y de dinero en relación con la disponibilidad económica para el ocio; días de juego en relación con los días sin juego); b) relación social (juego solitario o juego en compañía); c) motivación (jugar para divertirse, intentar ganar un dinero extra e interactuar con amigos o, por el contrario, jugar para evadirse de un estado de ánimo negativo, solucionar problemas económicos o hacer frente a deudas); d) interferencia en la vida cotidiana (inexistente o significativa); e) control sobre el juego (capacidad para ponerle freno cuando es oportuno o, por el contrario, gastar más dinero de lo planeado, ser incapaz de parar de jugar o apostar para recuperar lo perdido); y f) pensamientos sobre el juego (realistas y limitados al momento de jugar o distorsionados y omnipresentes incluso cuando el sujeto se ocupa de sus obligaciones cotidianas) (Blaszcczynski, Ladouceur y Shaffer, 2004).

En cuanto al tipo de pacientes indicados, no se ha conseguido establecer de forma operativa quiénes pueden beneficiarse específicamente de los programas de juego controlado. El grupo de Ladouceur (Ladouceur, 2005; Ladouceur, Sylvain, Boutin, Lachance, Doucet y Leblond, 2003) da a elegir a los pacientes la meta del tratamiento (abstinencia o control del juego), pero este enfoque resulta poco riguroso. A raíz de los conocimientos acumulados en el campo del abuso de alcohol, parece más razonable pensar que el juego controlado sea un objetivo válido para ciertos pacientes (los jugadores problemáticos, a quienes se puede motivar más fácilmente con esta propuesta), pero no para otros (los ludópatas propiamente dichos). Esto, en cualquier caso, requiere estudios ulteriores. De este modo, se podría contar con una caja de herramientas terapéutica, en donde las intervenciones breves con la meta del control del juego pueden inducir al tratamiento, facilitar la disposición para el cambio y reducir el riesgo de dependencia en jugadores problemáticos y en jugadores más jóvenes. A su vez, los programas de abstinencia pueden ser una alternativa específica para los ludópatas y para los pacientes que han fracasado en un programa de juego controlado. En este último caso se puede contar con una motivación adicional: la conciencia adquirida de que el establecimiento de límites en el juego no es una alternativa válida en su caso.

\section{Tratamientos psicológicos efectivos}

Al haberse considerado la ludopatía como una adicción, se ha recurrido a la utilización de técnicas terapéuticas que han mostrado su utilidad en el control del alcoholismo y de la drogadicción. De hecho, los primeros programas de tratamiento estructurados han sido en régimen de internamiento y comunes a los llevados a cabo con alcohólicos (McCormick y Ramírez, 1988). Actualmente la mayor parte de los programas de tratamiento se llevan a cabo en régimen ambulatorio e incluyen diversas alternativas (tratamiento psicológico, farmacoterapia, grupos de autoayuda) y diferentes formatos (individual o grupal). El internamiento en un centro hospitalario se limita a los casos con crisis agudas severas, con fracasos terapéuticos reiterados y con trastornos comórbidos, especialmente depresión e intentos de suicidio.

Las técnicas mejor estudiadas hasta la fecha son la desensibilización imaginada (variante de la desensibilización sistemática en la que las escenas no se presentan en un orden jerárquico), la reestructuración cognitiva, la terapia motivacional y la exposición en vivo con prevención de respuesta, junto con el control de estímulos. Los grupos de autoayuda, y especialmente Jugadores Anónimos (JA), han sido objeto también de diversos estudios (tabla 2). 
Tabla 2. Tratamientos principales del juego patológico

\begin{tabular}{ll}
\hline Tratamientos & Técnicas utilizadas \\
\hline Tratamientos conductuales & $\begin{array}{l}\text { Desensibilización imaginada } \\
\text { Relajación } \\
\text { Control de estímulos } \\
\text { Exposición en vivo con prevención de respuesta }\end{array}$ \\
\hline Tratamientos cognitivos & $\begin{array}{l}\text { Reestructuración cognitiva } \\
\text { Prevención de recaídas }\end{array}$ \\
& Terapia motivacional \\
\hline Tratamientos farmacológicos & $\begin{array}{l}\text { Antidepresivos IRSS } \\
\text { Antagonistas de los opiáceos } \\
\text { Estabilizadores del estado de ánimo } \\
\text { Neurolépticos atípicos }\end{array}$ \\
\hline Grupos de autoayuda & Jugadores Anónimos \\
\hline
\end{tabular}

\section{Terapias conductuales}

El enfoque conductual analiza la conducta de juego a partir de los antecedentes (expectativas económicas, señales de juego, emociones positivas o negativas, relaciones interpersonales, ansia de juego), las conductas abiertas o encubiertas (el dinero invertido en el juego, las estrategias de afrontamiento del malestar emocional, los pensamientos relacionados con el juego) y las consecuencias, tanto positivas (dinero ganado, activación psicofisiológica, oportunidades de socialización, evitación de la soledad) como negativas (deudas, depresión, baja autoestima, conflictos familiares y laborales, problemas con la Justicia). La activación fisiológica generada por el juego, el reforzamiento variable y el alivio de la tensión son los principales factores en el proceso de reforzamiento (Hodgins y Holub, 2007).

\section{a) Desensibilización imaginada y relajación}

La utilización de técnicas reductoras de ansiedad -la desensibilización imaginada, principalmente- está estrechamente relacionada con el "mecanismo de ejecución conductual" como teoría explicativa del mantenimiento del juego. Según esta teoría, en el Sistema Nervioso Central se establece un mecanismo neurofisiológico de ejecución de una conducta cuando dicha conducta se convierte en habitual. Este mecanismo es activado cuando la persona está en presencia de estímulos que preceden a la realización de la conducta habitual o cuando la persona piensa en llevar a cabo dicho comportamiento. Si la cadena de conductas no es completada cuando el sujeto es estimulado para llevarla a cabo, el mecanismo de ejecución conductual produce un aumento de activación y, por tanto, de tensión subjetiva. El malestar producido por esta situación fuerza al sujeto a completar la conducta, incluso aunque no desee hacerlo (McConaghy, Blaszczynski y Frankova, 1991).
La desensibilización imaginada implica que los sujetos, en primer lugar, tienen que describir unas cuantas situaciones que les impulsan a jugar, pero sin culminar en la conducta de juego. A continuación, se les enseña a tranquilizarse con un método breve de relajación. Después, los sujetos, en estado de relajación, tienen que imaginarse la primera situación, y así sucesivamente hasta completar las escenas descritas.

En el estudio de seguimiento a largo plazo de McConaghy et al. (1991), se comprueba la potencialidad terapéutica de la desensibilización imaginada, especialmente desde la perspectiva de costes y beneficios, que se muestra superior incluso a otras técnicas conductuales, como las técnicas aversivas, la relajación y la exposición en vivo. Lo más interesante de este estudio es que un programa terapéutico que implica a un único terapeuta, que dura solo 1 semana y que no requiere más de 14 horas de tratamiento, pueda tener una tasa de éxito global cercana al $66 \%$ de los casos tratados al cabo de 5 años de seguimiento. Resulta, sin embargo, cuestionable el hecho de llevarlo a cabo en régimen de internamiento, ya que la sencillez de la intervención terapéutica permite ejecutarla en un ámbito ambulatorio. Por ello, los pacientes elegidos, que están necesitados de una buena motivación para el internamiento, pueden no ser representativos de los pacientes que demandan ayuda terapéutica habitualmente.

b) Control de estímulos y exposición en vivo con prevención de respuesta

El control estimular implica limitar el uso del dinero del paciente (dinero en efectivo y tarjetas de crédito), impedir el recorrido de los circuitos de riesgo y evitar el contacto con amigos jugadores problemáticos. Una estrategia adicional es firmar una hoja de autoexclusión de los casinos y bingos, cancelar las tarjetas de crédito y transferir el control del dinero a otra persona. A medida que el tratamiento avanza, se atenúa el 
control estimular (excepto en lo que se refiere al contacto con los jugadores problemáticos y al programa de autoexclusión) para facilitar el autocontrol de la conducta de juego por parte del propio paciente. El programa de autoexclusión puede facilitar el compromiso de los pacientes con el cambio de conducta (Nelson, Kleschinsky, LaBrie, Kaplan y Shaffer, 2010).

A su vez, la exposición con prevención de respuesta tiene como objetivo provocar en los pacientes las ganas de jugar, exponiéndoles a estímulos que han sido suscitadores de deseo con anterioridad, y enseñarles a resistirlo en un programa de autocontrol progresivo. El objetivo de esta técnica es debilitar el poder de los estímulos para inducir el ansia y las conductas de juego, así como mostrar a los pacientes estrategias alternativas para hacer frente al malestar emocional suscitado. Con la repetición de la exposición sistemática a las situaciones de riesgo estas pierden la potencia de inducción al juego. Las tareas de exposición se llevan a cabo de forma regular (6 días por semana) en un entorno lo más similar a la vida real, a efectos de favorecer la generalización de estímulos y respuestas (tabla 3).

Tabla 3. Programa de exposición en vivo en el juego patológico

\begin{tabular}{l|l}
\hline Exposición & Características \\
\hline $1^{\text {a }}$ semana de exposición & $\begin{array}{l}\text { El coterapeuta (un familiar o amigo) está junto con el paciente cuando este practica los ejercicios de ex- } \\
\text { posición delante de una máquina de juego. } \\
\text { El paciente lleva el dinero justo para sus necesidades diarias. } \\
\text { El coterapeuta acude con el paciente al lugar de juego, pero permanece fuera, esperándole, mientras el } \\
\text { paciente practica los ejercicios de exposición. }\end{array}$ \\
& $\begin{array}{l}\text { El paciente lleva el dinero justo para sus necesidades diarias. } \\
\text { El coterapeuta permanece en casa cuando el paciente acude al lugar de juego para practicar los ejercicios } \\
\text { de exposición. Si el paciente se encuentra en un apuro, puede llamarle al móvil del coterapeuta, que está } \\
\text { disponible. }\end{array}$ \\
& $\begin{array}{l}\text { El paciente lleva el dinero justo para sus necesidades diarias. } \\
\text { El coterapeuta ya no forma parte de los ejercicios de exposición. } \\
4^{\text {a }} \text { semana de exposición }\end{array}$ \\
\hline
\end{tabular}

En el estudio de Echeburúa, Báez y Fernández-Montalvo (1996) se compara la eficacia diferencial de tres modalidades terapéuticas con el objetivo de la abstinencia del juego: a) tratamiento individual de control de estímulos y exposición en vivo con prevención de respuesta; b) terapia de grupo cognitivo-conductual; y c) una combinación de ambas modalidades terapéuticas. La importancia de este estudio radica en el tamaño de la muestra (64 jugadores patológicos), en la homogeneidad del juego (máquinas tragaperras), en la brevedad del tratamiento ( 6 semanas) y en que, por primera vez, se utiliza un grupo de control sin tratamiento con el que se pueden comparar los resultados de los grupos terapéuticos. En el seguimiento de los 12 meses los resultados más favorables correspondieron al primer grupo, en donde se obtuvo una tasa de éxito terapéutico del $68.8 \%$ frente al $37.5 \%$ de los grupos segundo y tercero. La terapia conductual se mostró más efectiva que la reestructuración cognitiva e incluso que la terapia combinada en relación con la conducta de juego.

Se ha llevado a cabo recientemente (Echeburúa, Gómez y Freixa, 2011) un estudio sobre el tratamiento del juego en 44 personas afectadas por esquizofrenia crónica y ludopatía. Se han comparado dos grupos: a) el grupo experimental $(n=23)$ (tratamiento farmacológico estándar para la esquizofrenia y programa cognitivo-conductual para el juego, centrado especialmente en el control de estímulos y la exposición gradual en vivo con prevención de respuesta, de 20 sesiones de duración); y b) el grupo de control $(n=21)$ (tratamiento farmacológico estándar para la esquizofrenia, sin tratamiento para el juego). Mientras los pacientes tratados en el grupo experimental mostraron una tasa de éxito del $73.9 \%$, solo el $19 \%$ de los participantes del grupo de control dejaron de jugar en el seguimiento de los 3 meses. Sin embargo, la mejoría del grupo experimental, aun siendo significativa, se redujo en el seguimiento de los 12 meses.

A diferencia de los estudios anteriores, en algunos trabajos, como el de Jiménez-Murcia et al. (2012), se concluye que la adición de la exposición y prevención de respuesta (separadas del control de estímulos) a la terapia cognitivo-conductual grupal aumenta las tasas de abandono. Falta por confirmar este aspecto en estudios posteriores.

En resumen, de acuerdo con el meta-análisis de Pallesen, Mitsem, Kvale, Johnsen y Molde (2005), los tratamientos centrados en el control de la ansiedad y la exposición a los estímulos de juego son efectivos para reducir el ansia de juego, el dinero apostado y el tiempo invertido en el juego, así como para lograr la abstinencia. Es más, estos cambios conductuales facilitan la modificación de las distorsiones cognitivas, sin llevar a cabo una intervención propiamente cognitiva. 


\section{Terapias cognitivas}

En los jugadores patológicos se detectan sesgos cognitivos que facilitan la implicación excesiva en el juego, tales como la ilusión de control, el pensamiento supersticioso o la percepción errónea del cálculo de probabilidades (la falacia del jugador). De este modo, la familiaridad con el juego fomenta la ilusión de control por parte del sujeto, así como la autoeficacia percibida en relación con la mayor probabilidad de ganancias. De ahí que los jugadores regulares tengan más pensamientos irracionales que los jugadores ocasionales, independientemente del tipo de juego, y que asuman más conductas de riesgo. Es decir, la regularidad de participación en el juego erosiona la racionalidad y facilita el establecimiento de un tipo de pensamiento irracional.

Por otra parte, los jugadores patológicos tienden a evaluar los resultados del juego de una manera sesgada, con la creencia de que las pérdidas continuadas anuncian la llegada de una ganancia inminente que les va a permitir recuperarse e incluso ganar. Es eso lo que les permite persistir en el juego a pesar de las persistentes pérdidas (Ladouceur, 2005).

Las terapias cognitivas ponen el énfasis en la modificación de las distorsiones cognitivas para reducir las ganas de jugar y las conductas de juego. El objetivo de las terapias cognitivas es enseñar al paciente a hacer frente a estos pensamientos irracionales y a establecer una relación adecuada entre los pensamientos, las emociones y las conductas, así como a detectar las autoverbalizaciones utilizadas en relación con el juego. En estas terapias también se adiestra al paciente a superar el ansia de juego y a controlar adecuadamente las emociones y se le enseñan técnicas de solución de problemas.

En las investigaciones clínicas más recientes con el juego patológico se han combinado técnicas conductuales y cognitivas (TCC) (corrección cognitiva de la percepción errónea de la probabilidad de ganar, entrenamiento en solución de problemas y entrenamiento en habilidades sociales). Así, el grupo de Ladouceur, en dos ensayos clínicos diferentes (Ladouceur et al., 2001, 2003) referidos al objetivo terapéutico del juego controlado, ha comparado la TCC (10 sesiones) en un formato individual con un seguimiento de 12 meses y la TCC (10 sesiones) en un formato grupal con un seguimiento de 24 meses, respectivamente, con un grupo de control de lista de espera. En el segundo ensayo se incorporó a la TCC un componente específico de prevención de recaídas. Los sujetos tratados en los grupos experimentales obtuvieron una mayor mejoría ( $85 \%$ y $88 \%$, respectivamente) respecto a los de los grupos de control (20\%) en cuanto a la reducción de los problemas con el juego. No obstante, en estos estudios el nivel de abandonos es muy alto (el $47 \%$ de los pacientes tratados en el primer estudio y el $26 \%$ en el segundo), lo cual rebaja considerablemente el porcentaje de éxito señalado por los autores.

También se ha comparado la TCC en un formato manualizado individual de 8 sesiones (dirigida por un psicólogo o completada por el propio paciente con un texto de autoayuda) con un grupo de pacientes de Jugadores Anónimos (JA). Los pacientes en todos los casos redujeron las conductas de juego, pero los resultados de la TCC en ambas variantes fueron superiores a los obtenidos en el grupo de JA en relación con la gravedad y la frecuencia de las conductas de juego (Petry et al., 2006).

En un estudio reciente de Grant, Donahue, Odlaug, Kim, Miller y Petry (2009) se comparó un programa cognitivoconductual (exposición en imágenes a los estímulos suscitadores de deseo e inducción de un estado de ánimo negativo, centrado en el pensamiento de las consecuencias negativas del juego, cuando el ansia de juego está activa, lo que es una forma de sensibilización encubierta) de 6 sesiones, con un programa de JA. Al cabo de 1 mes de seguimiento, el 64\% de los sujetos del programa cognitivo-conductual estaban abstinentes, pero solo lo estaban un $17 \%$ de los sujetos de JA.

En resumen, la TCC parece efectiva para reducir las conductas de juego. Cuando se aplica en un formato individual, la retención en el programa ha aumentado cuando se refuerza explícitamente por lo conseguido, se hacen llamadas recordatorias de las sesiones y se establece un diario de actividades que se revisa semanalmente. A su vez, los tratamientos grupales se refuerzan cuando cada paciente lleva a cabo tareas escritas individualizadas, de modo que se permite hacer un tratamiento a la medida de las necesidades del paciente (Melville, Davis, Matzenbacher y Clayborne, 2004). Sin embargo, aún no se ha conseguido establecer con precisión el número óptimo de sesiones necesario para reducir el juego y mantener la mejoría con el paso del tiempo (Grant y Odlaug, 2012).

\section{Terapia motivacional}

Otra alternativa terapéutica utilizada con los jugadores problemáticos es la terapia motivacional breve, basada en el apoyo telefónico, los manuales de autoayuda y los recursos on-line. La entrevista motivacional está centrada en detectar los puntos fuertes del paciente y en promover la motivación intrínseca para el cambio con el objetivo de que, al hacerle al sujeto consciente de que son mayores los inconvenientes que las ventajas de proseguir con este estilo de vida, el paciente resuelva la ambivalencia y se comprometa activamente con el cambio de conducta. 
Se trata de una terapia breve, que, a diferencia de las terapias tradicionales (que duran de 6 a 20 sesiones), cuenta con un contacto cara a cara con el terapeuta de solo 1 a 4 sesiones. La terapia motivacional puede ser una innovación para ayudar a los jugadores problemáticos que se niegan a buscar ayuda terapéutica en los recursos terapéuticos tradicionales y aumenta la percepción de control del paciente sobre su propia recuperación (Hodgins y Holub, 2007).

Este enfoque se ha mostrado efectivo, al menos con pacientes con problemas de juego menos severos. En el estudio de Hodgins, Currie y el-Guebaly(2001), con un seguimiento de 24 meses, hubo un $77 \%$ de la muestra que experimentó una mejoría en las conductas de juego y en el gasto de dinero. En general, el contacto personal, bien en forma de entrevista motivacional o bien en forma de apoyo telefónico, tiende a potenciar los resultados terapéuticos de los manuales de autoayuda (Hodgins, Currie, Currie y Fick, 2009).

La entrevista motivacional puede reforzar a la TCC en la reducción de las conductas de juego, incluso cuando se presenta en un formato on-line (Carlbring y Smith, 2006), así como en la reducción de las tasas de abandono del tratamiento (Wulfert, Blanchard, Freidenberg y Martell, 2006).

Sin embargo, la terapia motivacional, al margen de que resulte atractiva para los jugadores y de que puede facilitar la búsqueda de ayuda terapéutica, puede no ser tan efectiva con jugadores patológicos graves o que presentan un trastorno comórbido.

\section{Jugadores Anónimos (JA)}

Inspirado en la tradición y los principios espirituales de Alcohólicos Anónimos, JA desarrolla un programa de tratamiento grupal de autoayuda basado en la abstinencia como objetivo terapéutico. JA considera al juego patológico como una enfermedad que puede ser detenida, pero nunca curada. Los sujetos afectados van a tener siempre una predisposición a perder el control en el juego si de nuevo entran en contacto con él.

Con la ayuda de los doce pasos los jugadores pueden recuperarse y lograr la abstinencia. El objetivo del grupo es compartir intereses comunes, dar un marco de comprensión para lo que ocurre a sus componentes y dotar a todos ellos de un apoyo emocional, incluso espiritual, y de esperanza ante el futuro. El anonimato facilita la apertura a los miembros del grupo (Hodgins y Holub, 2007).

No hay datos inequívocos acerca de la eficacia de JA en estudios controlados y los estudios resultan contradictorios. Las tasas de recaídas tienden a ser altas. Según el estudio de Stewart y Brown (1988), la abstinencia se mantiene en solo un $7.5 \%$ de los sujetos evaluados un año después de la primera reunión. Asimismo la tasa de abandonos es alta. Sin embargo, en el estudio de Petry et al. (2006) los sujetos que acuden a JA obtienen mejores resultados que los que no lo hacen, estén o no en un tratamiento profesional al mismo tiempo (Petry et al., 2006). A su vez, en un estudio reciente de Toneatto y Dragonetti (2008) no hubo diferencias entre la TCC y la terapia de JA ni en la tasa de abstinencia ni en el dinero gastado en el juego a los 12 meses de seguimiento.

Se está estudiando también la efectividad terapéutica de JA en relación con la participación de las parejas de los jugadores en grupos de familiares. Al parecer, las tasas de abstinencia de los jugadores son mayores cuando sus parejas acuden a los grupos de familiares.

JA es muy popular, pero se necesita más investigación para determinar su papel en la recuperación de los jugadores y para reducir las tasas de abandono existentes, así como para precisar el perfil de los jugadores que pueden beneficiarse de esta modalidad terapéutica.

\section{Prevención de recaídas}

Las recaídas constituyen un desafío en el tratamiento de todas las adicciones porque son muy frecuentes y suelen tener lugar en los primeros meses tras la terminación del tratamiento. En el juego patológico las situaciones precipitantes de la recaída, según el estudio de Echeburúa, Fernández-Montalvo y Báez (2001), han resultado ser, por orden de importancia, las siguientes: el manejo inadecuado del dinero, los estados emocionales negativos, el consumo abusivo de alcohol, el ansia de juego y, por último, la presión social. En concreto, casi la mitad de todas las recaídas se han producido por un control inapropiado del dinero (disponibilidad de unos ingresos económicos inesperados, vigilancia excesiva del dinero por parte de la familia, terminación del pago de las deudas, etcétera). El segundo factor en importancia ha sido la presencia de estados emocionales negativos (aburrimiento/tristeza, ansiedad/estrés, ira/ discusiones, etcétera), que explica el $25 \%$ de las recaídas. El resto de categorías (consumo abusivo de alcohol, ansia de jugar y presión social), si bien son también importantes, explican un porcentaje menor de los fracasos terapéuticos.

La prevención de recaídas está centrada en dotar de habilidades al paciente para identificar las situaciones de alto riesgo, tales como los estados emocionales negativos, los conflictos interpersonales y la presión social, y en ayudarle a hacerles frente con estrategias de afrontamiento adecuadas. El enfoque de la solución de problemas tiene 
como objetivo abordar la impulsividad y el ansia de juego y resolver dificultades emocionales para las que el juego constituye un escape. Una recuperación duradera implica asimismo la adquisición de un nuevo estilo de vida sano que promueva conductas de ocio e interpersonales incompatibles con la adicción (Marlatt y Gordon, 2005).

De este modo, se han desarrollado algunos programas específicos para la prevención de recaídas en el ámbito del juego patológico. Así, por ejemplo, en el ensayo clínico de Echeburúa, Fernández-Montalvo y Báez (2000) se puso a prueba la efectividad de dos modalidades de prevención de recaídas -grupal e individual-, basadas en el modelo de Marlatt y Gordon, en relación con un grupo de control. Para ello, se utilizó una muestra de 69 jugadores, tratados todos ellos, en una fase previa, mediante exposición en vivo con prevención de respuesta y control de estímulos. Posteriormente, una vez conseguida la abstinencia, recibían la intervención en prevención de recaídas solo los sujetos de los dos grupos experimentales. Los resultados obtenidos en el seguimiento de los 12 meses mostraron la superioridad de los grupos terapéuticos en relación con el grupo de control. En concreto, en este seguimiento los dos grupos terapéuticos eran homogéneos entre sí, con una tasa de éxitos del $82 \%$ en la modalidad individual y del $78 \%$ en la grupal, y significativamente superiores al grupo de control (52\%). La tasa de abandonos fue del $14 \%$. Una descripción detallada de la guía de tratamiento utilizada para la prevención de recaídas, con el diario de sesiones correspondiente y los instrumentos de evaluación empleados, puede encontrarse en Fernández-Montalvo y Echeburúa (1997).

\section{Terapia farmacológica}

El interés de la terapia farmacológica en el juego patológico deriva del papel desempeñado por algunos neurotransmisores, como la serotonina, la norepinefrina y la dopamina, en los mecanismos de recompensa cerebral. En concreto, la norepinefrina se ha asociado a la activación y a la búsqueda de novedades, la dopamina al sistema de recompensas y a la motivación y, finalmente, la serotonina a la impulsividad y la compulsividad (Hollander, Sood, Pallanti, Baldini-Rossi y Baker, 2005).

Los fármacos puestos a prueba con el juego patológico, que actúan sobre alguno de estos sistemas de neurotransmisión, son los antidepresivos inhibidores de la recaptación de la serotonina (IRSS), los estabilizadores del estado de ánimo, los neurolépticos atípicos y los antagonistas opiáceos.
En concreto, los antidepresivos IRSS se han mostrado útiles para reducir el ansia de juego o impedir las conductas impulsivas/compulsivas o cuando existe una comorbilidad con los trastornos de ansiedad. No obstante, los déficits de serotonina de los jugadores patológicos pueden ser causa, pero también consecuencia del trastorno. En este supuesto solo se estaría tratando un síntoma parcial del juego. Además, la baja proporción de serotonina también está asociada a otros síntomas clínicos que aparecen en el juego patológico (ansiedad, depresión, ideación suicida, etcétera). Así, cabe preguntarse si la alteración bioquímica referida se asocia en realidad al juego patológico o más bien a los síntomas psicopatológicos que con frecuencia lo acompañan.

A su vez, los antagonistas opiáceos, como la naltrexona, pueden bloquear los sentimientos de euforia relacionados con el juego, lo que puede reducir el ansia y los pensamientos de juego y mejorar el funcionamiento psicosocial, y están indicados cuando el ansia de juego al comienzo del tratamiento es muy fuerte y cuando hay una historia familiar de alcoholismo. Por último, los estabilizadores del estado de ánimo, tales como el litio o el topiramato, pueden ser útiles en el tratamiento del juego cuando este se utiliza para regular estados de ánimo negativos. En cualquier caso, el apoyo empírico es aún reducido (Potenza, 2012).

A nivel clínico, los psicofármacos tienen utilidad cuando hay una comorbilidad del juego patológico con otros trastornos, tales como la depresión, el trastorno bipolar o el trastorno de déficit de atención con hiperactividad.

En resumen, los fármacos pueden ser beneficiosos como un tratamiento complementario a la terapia psicológica en determinadas condiciones. Se han puesto a prueba distintos psicofármacos para el tratamiento del juego patológico, pero todavía no existen medicaciones aprobadas específicamente para la terapia de este trastorno. Por otra parte, las tasas de abandono con los psicofármacos en este trastorno son altas (30\%-50\%) (Grant, Odlaug y Schreiber, 2012).

\section{Discusión}

Los estudios referidos a la terapia del juego patológico prueban que este cuadro clínico responde bien a los tratamientos ofertados. Desde una perspectiva general, se han obtenido unas tasas de éxito superiores al 50\% de los casos tratados. Además, esta tasa de éxito puede aumentar incluso hasta el $80 \%$ cuando se aplican técnicas conductuales -control de estímulos y exposición en vivo con prevención de respuesta, principalmente- seguidas de una intervención cognitivo-conductual en prevención 
de recaídas (Echeburúa et al., 2000; Pallesen et al., 2005; Toneatto y Ladouceur, 2003).

Sin embargo, solo un $10 \%$ de los jugadores patológicos en la comunidad buscan ayuda terapéutica (Ladouceur, 2005). Atraer a los pacientes al tratamiento es un reto terapéutico importante. Si bien es cierto que hay en torno a un $35 \%$ de los sujetos con problemas de juego que pueden experimentar una remisión espontánea, la mayor parte de los pacientes muestran un curso crónico y una fluctuación en la severidad de los síntomas con el paso del tiempo, que hace necesaria una ayuda profesional (Grant y Odlaug, 2012).

La adherencia al tratamiento es un desafío para la investigación futura. La ambivalencia de muchos pacientes respecto a su disposición a dejar de jugar y a modificar su tipo de vida explica la alta tasa de abandonos (15\%-30\%). Si bien no hay soluciones mágicas para resolver este problema, la terapia motivacional, los contractos conductuales y los objetivos terapéuticos flexibles (juego controlado o abstinencia) pueden facilitar la adherencia al tratamiento. Por ello, se requiere más información acerca de cuándo establecer un programa de abstinencia o de juego controlado (Echeburúa y Corral, 2008; Ladouceur, Lachance y Fournier, 2009).

Las terapias psicológicas deben ser adaptadas a las necesidades específicas de cada paciente en función del tipo de juego, del sexo, de la presencia de trastornos comórbidos, etcétera. Los jugadores patológicos no constituyen un grupo homogéneo y no pueden ser tratados, por tanto, de una forma única. Todavía se ha avanzado poco en esta dirección (Echeburúa, González-Ortega, Corral y Polo-López, 2013).

Se ha iniciado un enfoque nuevo por medio del tratamiento de los familiares que conviven con un jugador patológico. Así, se ha comenzado a desarrollar el modelo de "Community Reinforcement and Family Therapy" (CRAFT), centrado en la enseñanza a los familiares de principios conductuales para reforzar las conductas incompatibles con el juego (Hodgins, Toneatto, Makarchuk, Skinner y Vincent (2007), o programas de entrenamiento en habilidades de afrontamiento para familiares que tienen un jugador patológico (Rychtarik y McGillicuddy, 2006).

Si bien no se cuenta aún con un tratamiento basado en la evidencia y es, por ello, prematuro obtener conclusiones definitivas, las terapias conductuales y cognitivo-conductuales en sus diferentes formatos (individual, grupal, autoayuda) constituyen la primera línea de tratamiento del juego patológico (Gooding y Tarrier, 2009). Por mucho que estén presentes alteraciones bioquímicas, no se pueden soslayar los importantes factores de aprendizaje, de control de estímulos, de distorsiones cognitivas, etcétera, que contribuyen a mantener las conductas de juego. A modo complementario, el tratamiento farmacológico, sobre todo con antidepresivos IRSS y antagonistas de los opiáceos, puede estar indicado solo en aquellos casos de ludopatía con depresión grave y fuerte ansiedad, ideación suicida y/o conductas impulsivas descontroladas. A su vez, el régimen de internamiento puede ser adecuado para jugadores patológicos severos, con trastornos comórbidos o ideación suicida grave (Grant y Odlaug, 2012).

Todavía quedan puntos por esclarecer respecto a las terapias conductuales y cognitivas. No se conoce cuál de las terapias propuestas es la más idónea (o para qué tipo de pacientes) ni cuál es la duración óptima de la terapia. Asimismo hay que delimitar el perfil de los pacientes que pueden beneficiarse de la terapia en un formato individual, grupal o de autoayuda (Oei, Raylu y Casey, 2010).

Finalmente, el juego en Internet (por ejemplo, el póquer on-line, los casinos y bingos on-line o las casas de apuestas en Internet), en la medida en que facilita el anonimato, es accesible en cualquier momento y lugar y está al alcance de los menores, plantea una serie de retos nuevos y requiere una reacomodación de las terapias actualmente disponibles (Derevenski y Gupta, 2007; Griffiths y Barnes, 2008; Laplante y Braverman, 2010).

\section{Referencias}

American Psychiatric Association (2013). Diagnostic and statistical manual of mental disorders ( $5^{\text {th }}$ edition). Washington, DC: Author.

Becoña, E. (1999). Epidemiología del juego patológico en España. Anuario de Psicología, 30,7-19.

Blaszczynski, A., Ladouceur, R., y Shaffer, H. H. (2004). A science-based framework for responsable gambling: The Reno model. Journal of Gambling Studies, 20, 301-317.

Carlbring, P., y Smith, F. (2008). Randomized trial of Internet-delivered self-help with telephone support for pathological gamblers. Journal of Consulting and Clinical Psychology, 76, 1090-1094.

Derevensky, J. L., y Gupta, R. (2007). Adolescent Gambling: Current Knowledge, Myths, Assessment Strategies, and Public Policy Implications. In G. Smith, D.C. Hodgins y R. J. Williams (eds), Research and Measurement Issues in Gambling Studies (pp. 437-463). Elsevier: Amsterdam.

Echeburúa, E., y Corral, P. (2008). Juego responsable: ¿una alternativa para la prevención y el tratamiento de la ludopatía? Adicciones, 20,321-326.

Echeburúa, E., Báez, C., y Fernández-Montalvo, J. (1996). Comparative effectiveness of three therapeutic modalities in the psychological treatment of pathological gambling: long-term outcome. Behavioural and Cognitive Psychotherapy, 24,51-72.

Echeburúa, E., Fernández-Montalvo, J., y Báez, C. (2000). Relapse prevention in the treatment of pathological gambling: long-term outcome. BehaviorTherapy, 31,351-364.

Echeburúa, E., Fernández-Montalvo, J., y Báez, C. (2001). Predictors of therapeutic failure in pathological gamblers following behavioural treatment. Behavioural and Cognitive Psychotherapy, 29, 369-373.

Echeburúa, E., Gómez, M., y Freixa, M. (2011). Cognitive-behavioural treatment of pathological gambling in individuals with chronic schizophrenia. A pilot study. Behaviour Research and Therapy, 49,808-814. 
Echeburúa, E., González-Ortega, I., Corral, P., y Polo-López, R. (2011). Clinical gender differences among adult pathological gamblers seeking treatment. Journal of Gambling Studies, 27,215-227.

Echeburúa, E., González-Ortega, I., Corral, P., y Polo-López, R. (2013). Pathological gamblers and a non-psychiatric control group taking gender differences into account. Spanish Journal of Psychology, 16, 1-9.

El-Guebaly, N., Mudry, T., Zohar, J., Tavares, H., y Potenza M. N. (2012). Compulsive features in behavioural addictions: the case of pathological gambling. Addiction, 107, 1726-1734.

Fernández-Montalvo, J., y Echeburúa, E. (1997). Manual del juego patológico. Ayuda para el paciente y guía para el terapeuta. Madrid: Pirámide.

González-Ortega, I., Echeburúa, E., Corral, P., Polo-López, R., y Alberich, S. (2013). Predictors of pathological gambling severity taking gender differences into account. European Addiction Research, 19,146-154.

Gooding, P., y Tarrier, N. (2009). A systematic review and meta-analysis of cognitive-behavioural interventions to reduce problem gambling: Hedgings our bets? Behaviour Research and Therapy, 47, 592-607.

Grant, J. E., y Odlaug, B. L. (2012). Psychosocial Interventions for Gambling Disorders. Increasing the Odds. What Clinicians Need to Know About Gambling Disorders (vol 7, pp. 38-51). National Center for Responsible Gaming: Washington, DC.

Grant, J. E., Odlaug, B. L., y Schreiber, L. N. (2012). Pharmacological Treatments in Pathological Gambling.British Journal of Clinical Pharmacology.doi: 10.111/j.1365-2125.2012.04457.x

Grant, J. E., Donahue, C. B., Odlaug, B. L., Kim, S.W., Miller, M. J., y Petry, N. M. (2009). Imaginal desensitization plus motivational interviewing gor pathological gambling: randomized controlled trial. British Journal of Psychiatry, 195, 266-267.

Griffiths, M. D., y Barnes, A. (2008). Internet gambling: An online empirical study among student gamblers. International Journal of Mental Health and Addiction, 6, 194-204.

Hodgings, D. C., y Holub, A. (2007). Treatment of Problem Gambling. In G. Smith, D.C. Hodgins y R.J. Williams (eds), Research and Measurement Issues in Gambling Studies (pp. 372-397). Elsevier: Amsterdam.

Hodgins, D. C., Currie, S. R., y el-Guebaly, N. (2001). Motivational enhancement and self-help treatments for problem gambling. Journal of Consulting and Clinical Psychology, 69, 50-57.

Hodgins, D. C., Currie, S. R., Currie, G., y Fick, G.H. (2009). Randomized trial of brief motivational treatments for pathological gamblers: More is not necessary better. Journal of Consulting and Clinical Psychology, 77, 950-960.

Hodgings, D. C., Toneatto, T., Makarchuk, K., Skinner, W., y Vincent, S. (2007). Minimal treatment approaches for concerned significant others of problem gamblers: A randomized controlled trial. Journal of Gambling Studies, 23, 215-230.

Hollander, E., Sood, E., Pallanti, S., Baldini-Rossi, N., y Baker, B. (2005). Pharmacological treatment of pathological gamblers.Journal of Gambling Studies, 21, 101-110.

Jiménez-Murcia, S., Aymaní, N., Gómez-Peña, M., ...Santamaría, J. J. (2012). Does exposure and response prevention improve the results of group CBT for male slot machine pathological gamblers? British Journal of Psychiatry, 51, 54-71.

Ladouceur, R. (2005). Controlled gambling for pathological gamblers. Journal of Gambling Studies, 21, 49-57.

Ladouceur, R., Lachance, S., y Fournier, P. M. (2009). Is control a viable goal in the treatment of pathological gambling? Behaviour Research and Therapy, 47, 189-197.
Ladouceur, R., Sylvain, C., Boutin, C., Lachance, S., Doucet, C., y Leblond, J. (2003). Group therapy for pathological gamblers: A cognitive approach.Behaviour Research and Therapy, 41,587-596.

Ladouceur, R., Sylvain, C., Boutin, C., Lachance, S., Doucet, C., Leblond, J., y Jacques, C. (2001). Cognitive treatment of pathological gambling.Journal of Nervous and Mental Disease, 189, 774-780.

Laplante, D. A., y Braverman, J. (2010). El juego en Internet: situación actual y propuestas para la prevención y la intervención. En E. Echeburúa, E. Becoña y F. J. Labrador (eds), El juego patológico. Avances en la clínica y en el tratamiento (pp. 323-358). Pirámide: Madrid.

Lorains, F. K., Cowlishaw, S., y Thomas, S. A. (2011). Prevalence of comorbid disorders in problem and pathological gambling: systematic review and meta-analysis of population surveys. Addiction, 106, 490-498.

Marlatt, G.A., y Gordon, J.R. (Eds.) (2005). Relapse prevention: Maintenance strategies in addictive behavior change ( $2^{\text {nd }} e d$.). New York: Guilford Press.

McConaghy, N., Blaszczynski, A., y Frankova, A. (1991). Comparison of imaginaldesensitisation with other behavioural treatments of pathological gambling. A two-to nine-year follow-up. British Journal of Psychiatry, 159, 390-393.

Melville, C. L., Davis, C. S., Matzenbacher, D. L., y Clayborne, J. (2004). Node-link-mapping-enhanced group treatment for pathological gambling. Addictive Behaviors, 29, 73-87.

McCormick, R. A., y Ramírez, L.F. (1988). Pathological gambling. In J.G. Howells (ed.). Modern perspectives in psychosocial pathology (pp. 45-57), New York: Brunner/Mazel Inc.

Nelson, S. E., Kleschinsky, J. H., LaBrie, R. A., Kaplan, S., y Shaffer, H. J. (2010). One decade of self exclusion: Missouri casino self-excluders four to ten years after enrolment. Journal of Gambling Studies, 26, 129-144.

Oei, T. P. S., Raylu, N., y Casey, L. (2010). The effectiveness of group versus individual CBT programs for problem gamblers: A randomized clinical trial. Behavioural and Cognitive Psychotherapy,38, 233-238.

Pallesen, S., Mitsem, M., Kvale, G., Johnsen, B. H., y Molde, H. (2005). Outcome of psychological treatments of pathological gambling: A review and meta-analysis. Addiction, 100, 1412-1422.

Petry, N. M., Ammerman, Y., Bohl, J., Doersch, A., ...Gay, H. (2006). Cognitive-behavioral therapy for pathological gamblers. Journal of Consulting and Clinical Psychology, 74, 555-567.

Potenza, M. N. (2012). Pharmacological Approaches to Treating Pathological Gambling.Increasing the Odds (vol 7). What Clinicians Need to Know About Gambling Disorders (vol 7, pp. 52-60). National Center for Responsible Gaming: Washington DC.

Rychtarik, R. G., y McGillicuddy, N. B. (2006). Preliminary evaluation of a coping skills training program for those with a pathological-gambling partner. Journal of Gambling Studies, 22, 165-178.

Stewart, R. M., y Brown, R. (1988). An outcome study of Gamblers Anonymous. British Journal of Psychiatry, 152, 284-288.

Toneatto, T., y Dragonetti, R. (2008). Effectiveness of community-based treatment for problem gambling: A quasi-experimental evaluation of cognitive-behavioral vs. twelve-step therapy. American Journal on Addictions, 17, 298-303.

Toneatto, T., y Ladouceur, R. (2003). Treatment of pathological gambling: A critical review of the literature. Psychology of Addictive Behaviors, 17,284-292.

Wulfert, E., Blanchard, E. B., Freidenberg, B. M., y Martell, R. S. (2006). Retaining pathological gamblers in CBT through motivational enhancement: A pilot study. BehaviorModification, 30, 315-340. 